\title{
SIRCAUR: SAFE INSPECTION OF REINFORCED CONCRETE STRUCTURES BY AUTONOMOUS ROBOT
}

\author{
GABRIELA GALLEGOS GARRIDO, MAHESH DISSANAYAKE and TARIQ SATTAR \\ London South Bank University, London South Bank Innovation Centre, \\ Granta Park, Great Abington, Cambridge CB21 6AL, UK. \\ gallegog@lsbu.ac.uk,dissanad@lsbu.ac.uk,sattartp@lsbu.ac.uk
}

\author{
ANGELOS PLASTROPOULOS \\ Innovative Technology and Science Limited, \\ Hildersham Road, Cambridge, Cambridgeshire CB21 6DR, UK \\ angelos.plastropoulos@innotecuk.com \\ MUNTASIR HASHIM \\ The Welding Institute Ltd. NDT group \\ Granta Park, Great Abington, Cambridge CB21 6AL, UK. \\ muntasir.hashim@twi.co.uk
}

\begin{abstract}
Designing climbing robots for industrial applications, has been proven to be an egg and chicken problem. The more the payload the more adhesion force needed. In order to move a robot upwards with such a force, motors with bigger torque are required and thus, increasing the payload. Similarly, increasing the adhesion force is likely to increase the payload. This paper presents an improved climbing robot specifically designed to climb on structures with 30 35mm of reinforced concrete (R/C) cover, while deploying a precision noncontact GPR sensor for the detection of rebar corrosion and related defects. Furthermore, it carries a system for visual inspection and detection of concrete cover deterioration. It works autonomously under UWB controlled trajectories, adjustable by GPR sensor feedback for avoidance of rebar-poor regions. The inspection NDT data is transmitted wirelessly to a ground-based CPU for processing and monitoring.
\end{abstract}

\section{Introduction}

The operating environment and the task to be carried out are the main factors to take into consideration when designing climbing robots for industrial applications. For climbing on nonferrous surfaces, systems tend to employ either energy intensive suction cups or dynamic vortex adhesion methods, but when it comes to ferrous surfaces, the preferred choices are either permanent magnets or electromagnetic adhesion. While electromagnets offer the ability to control the magnetic field on demand, they require constant energy consumption to maintain their magnetic field [3][4].

On the other hand, corrosion of materials and structures costs the UK economy typically 3.5\% of the gross domestic product (GDP) per annum [1], amounting to £100bnpa. Such a percentage means that improved methods of corrosion mitigation can make an impact on the economy as a whole. $\mathrm{R} / \mathrm{C}$ is the most common construction material used throughout the civil engineering and energy-producing sector for roads, bridges, high-rise buildings, dams, cooling towers, storage tanks, oil and gas lines, offshore energy platforms etc. $\mathrm{R} / \mathrm{C}$ accounts for about $8 \%$ of total global corrosion costs [2] and is £8bnpa in the UK alone. Currently, R/C inspection is carried out manually by a person positioned on expensive scaffolding or by abseiling, with this comes a high safety risk. SIRCAUR aims to tackle these problems by deploying a precision non-contact GPR sensor for the detection of rebar corrosion and related defects, with $100 \%$ volume coverage and no scaffolding or abseiling. The crawler has been successfully tested on an R/C wall with $\sim 30-35 \mathrm{~mm}$ of concrete cover and the NDT system was able to effectively detect defects. 
Previous research on wall-climbing robots has focused on the payload capacity, mobility adhesion safety and energy consumption [3] [4]. Mobile robot based concrete inspection techniques have been studied in [5] [6] [7]. The design, prototype development and testing of the SIRCAUR robot are illustrated in this paper.

\section{Design of the robot}

\subsection{Development of the adhesion module}

Mobility of a climbing robot is based on the locomotion and the adhesion principles. Therefore, a suitable adhesion mechanism was designed using COMSOL Multiphysics. Capabilities of the permanent magnetic adhesion for concrete climbing robots is discussed in [6]. Even though this problem has been studied previously, the engineering difficulty of reinforced concrete climbing remains a challenge, as it is necessary to balance the adhesion force with the payload of the robot. The greater the payload/weight of the robot, more adhesion force is needed. In order to enable the robot to move under such forces, the drive motors require significant torque, which in turn requires larger motors, increasing the overall payload.

The proposed adhesion module uses several permanent magnets and a flux concentrator called "yoke" to optimize the features of the adhesion module. The aim is to achieve the maximum adhesion force while keeping the module weight at the minimum. During the COMSOL optimisation process, physical parameters of the previously discussed magnet-yoke adhesion module [6] were changed. Different parameters such as the size, relative permeability of the magnet and the ferromagnetic structure were considered. As it was discussed in [6] [8], the air gap or distance between the ferrous surface and the magnet is an important parameter when designing an adhesion system for complex structures.

In [6] it has been analysed and tested the best configuration to achieve the maximum adhesion force, which is using three magnets with N-S-N orientation on an iron yoke. Thus, the new model is composed of an iron yoke $(250 \times 50 \times 10 \mathrm{~mm})$, three N52 rare earth neodymium magnets arranged on top of the yoke on a N-S-N configuration with $50 \mathrm{~mm}$ spacing (around $2.5 \mathrm{~kg}$ net weight). The magnetic adhesion system (yoke + magnets) was simulated over $12 \mathrm{~mm}$ steel rebar with $30 \mathrm{~mm}$ of concrete cover (air gap). The resulting adhesion force computed at $143.1 \mathrm{~N}$ (14.6kg).

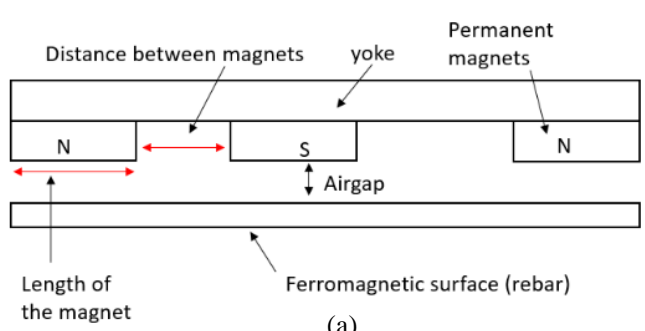

(a)

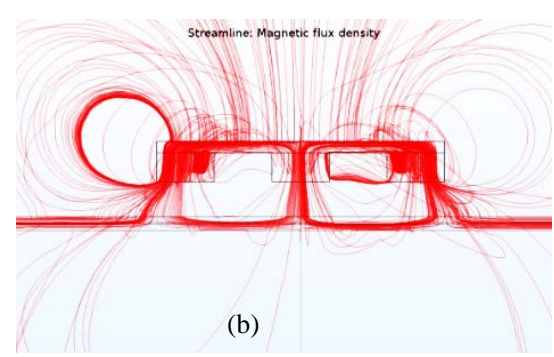

(b)

Figure 1: (a) magnet -yoke modelling layout. (b) Magnetic flux distribution.

Table 1: Material properties for COMSOL simulations.

\begin{tabular}{lc}
\hline \multicolumn{1}{c}{ Properties } & Value \\
\hline Magnetic induction intensity mag $\mathrm{Br}_{\mathrm{r}}(\mathrm{T})$ & 1.47 \\
\hline Magnetic Coercive force $\mathrm{H}_{\mathrm{cb}}(\mathrm{KA} / \mathrm{m})$ & 796 \\
\hline Intrinsic Coercive force $\mathrm{H}_{\mathrm{ci}}(\mathrm{KA} / \mathrm{m})$ & 876 \\
\hline Magnetic energy product $\mathrm{HB}\left(\mathrm{KJ} / \mathrm{m}^{3}\right)$ & 398 \\
\hline Relative permeability of magnets $\left(\mu_{\mathrm{r}}\right)$ & 1.05 \\
\hline Relative permeability of steel plate & 1000 \\
\hline Relative permeability of iron yoke & 4500 \\
\hline
\end{tabular}


Streamline plot show the magnetic flux behaviour as illustrated in figure 1 . In figure 1(b), the flux is clearly concentrated on the iron yoke and uniformly distributed through the air gap towards the iron rebar. The effect of the yoke, also called shielding, allows to safely mounting electronics devices on top of the system while avoiding interference. COMSOL modelling final parameters used are tabulated in table 1 . Test and validation are discussed in section 3 .

\subsection{Development of the inspection module}

Ground Penetrating Radar (GPR) is one of the most effective forms of Non-Destructive Testing (NDT) methods with a variety of applications on concrete structures. This technique is commercially available since 1970's. Consequently, numerous studies have been carried out to develop the use of GPR for a wide range of concrete applications. In particular, for the detection of different types of defects that can occur in rebars or overlaid reinforced concrete decks, measurement of the thickness of concrete members and void detection [9] [10]. This method of inspection was originally developed for mapping geological features and recently has been utilized in engineering. It offers a unique non-invasive and non-destructive way of characterizing the surface and subsurface features. However, while GPR does offer many advantages for deployment on a robot particularly its lightness $(<2 \mathrm{KG})$ and high scanning speed, it also has limitations. GPR cannot detect delamination, honeycombing, grouting defects or measure the quality variation of the concrete. Other methods are more effective for detecting the flaws which GPR cannot detect but they are impractical for deployment on a robot, either for the weight or because they can only be used in a static position.

The PicoR-2K GPR is a geophysical device designed for subsurface sensing and monitoring of opaque materials such as ice, snow, road pavement, soil, etc. This device enables calculation of the thickness of the layers inside such materials, as well as detection and positioning of inhomogeneities within the material. The PicoR-2K GPR evaluation kit (summary specification shown in Table 2), was employed for the defect sensitivity trials.

Table 2: PicoR-2K GRP summary specification.

\begin{tabular}{ll}
\hline Parameter & Value \\
\hline Frequency range (at a level of $-10 \mathrm{~dB})$ & $0.5-3.5 \mathrm{GHz}$ \\
\hline Central frequency & $1600 \mathrm{MHz}$ \\
\hline Pulse duration & $60 \mathrm{ps}$ \\
\hline Pulse amplitude voltage & $6 \mathrm{~V}$ \\
\hline Pulse repetition frequency & $50 \mathrm{MHz}$ \\
\hline Detection range / depth (with RVS-A1 UWB antennas): & \\
$\quad \quad$ Moving person in an open area & Up to $15-20 \mathrm{~m}$ \\
$\quad$ Moving person behind the wall & $\mathrm{Up}$ to $6-8 \mathrm{~m}$ \\
$\quad$ Measured ice thickness & $\mathrm{Up}$ to $15-2 \mathrm{~m}$ \\
$\quad$ Measured asphalt thickness & $\mathrm{Up}$ to $0.5 \mathrm{~m}$ \\
$\quad$ Detection of objects in the ground & $\mathrm{Up}$ to $0.5-1 \mathrm{~m}$ \\
\hline Range measurement accuracy & $1-2 \mathrm{~cm}$ \\
\hline
\end{tabular}

\subsection{Development of the localization module and the robot structure}

Ultra-Wide Band (UWB) localization technique was chosen for this project due to the accuracy and the application feasibility (i.e. ability to work with complex geometries). Commercially available POZYX ${ }^{\mathrm{TM}}$ UWB module was selected after considering its compatibility with the existing operational microcontroller. During the design phase, the software for localization was developed and tested using an Arduino on an AmigoBot robot. Both were used to replicate the movements of the robotic platform before having the prototype. Furthermore, a ROS (Robot Operating System) node was developed so that the localization module can be incorporated into the robot's software architecture. 
Structure of the physical robot was designed to fit on to the concrete surfaces as illustrated in figure 2. The position of the adhesion module under the belly of the robot was designed to coincide with the rebars, which were buried under the climbing surface. Moreover, a design optimization was necessary in order to maintain the balance between robots' physical parameters such as motors, weight, payload and the strength of the adhesion module. Therefore, a series of computer aided design optimization tasks were conducted to build the platform architecture.

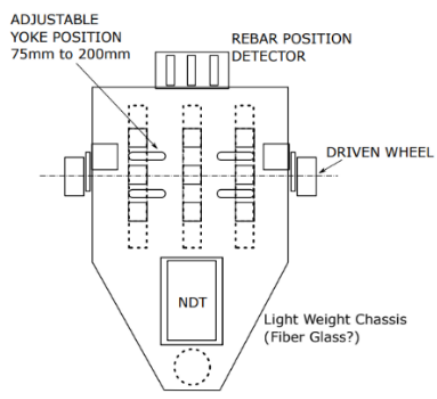

(a)

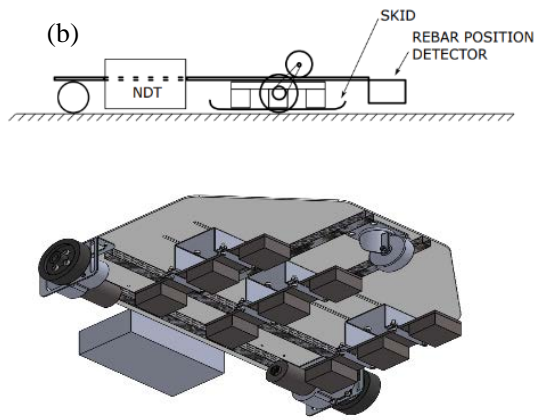

(c)

Figure 2: (a) and (b) Schematic of the robot. (c) CAD design sample.

\section{Prototype and testing}

\section{1. $\quad$ Adhesion module testing}

Prototype of the adhesion module was conducted according to the designed parameters obtained during the COMSOL numerical modelling phase. Special care was taken while measuring and positioning the magnets on the yoke to build the adhesion system (figure 6). The total weight of the adhesion system was $2.83 \mathrm{~kg}$, measured with an industrial measuring scale.
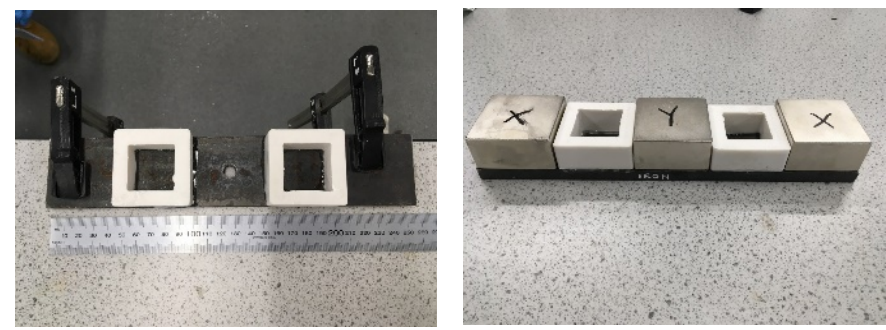

Figure 3: Construction of the magnetic adhesion system.

In order to validate the results of the numerical modelling results, an experiment was carried out using a load cell machine for force/tension testing, namely the Instron 5567A B723. In this study, calibration and assessment of the test machine was conducted in accordance with ISO 7500-1:2015 and using Instron procedure N001.The adhesion system on the load cell was set to a distance of $200 \mathrm{~mm}$ from the rebar (see figure 4). Data was recorded (6 values per second), while slowly approaching the system to the $12 \mathrm{~mm}$ rebar until a $20 \mathrm{~mm}$ spacing gap was left in between the magnets and the rebar. The experiment was repeated for $16 \mathrm{~mm}$ and $20 \mathrm{~mm}$ rebar diameters.

In order to abstract the experimental procedure, the following results have been performed with the consideration of a $12 \mathrm{~mm}$ rebar. Moreover, most of reinforced concrete structures are designed with the rebars within a distance of $30-35 \mathrm{~mm}$ (thickness of the concrete). Also, the maximum concrete cover over the rebars can reach up to $50 \mathrm{~mm}$. Therefore, the simulation was carried out for a 50mm concrete cover and obtained a magnetic force of 31.39N. 


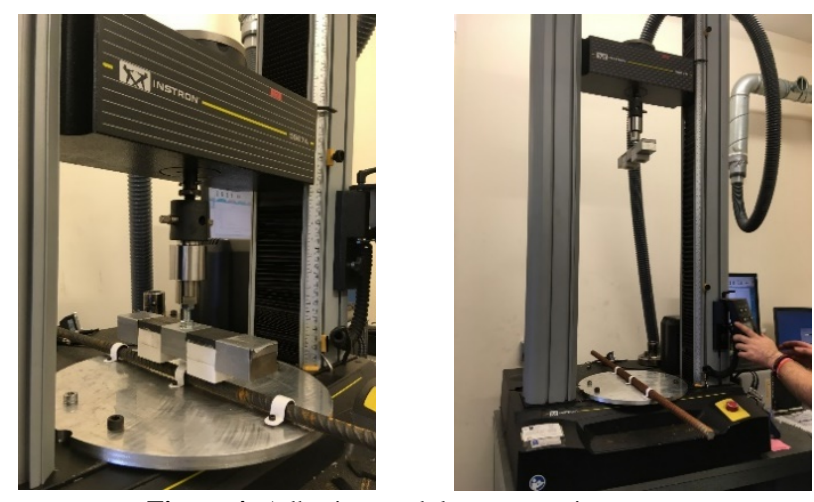

Figure 4: Adhesion module test experiment

As expected, the gap between the magnet surface and the climbing surface is critical, as a small gap increases the adhesion force significantly. The experiment was carried out by varying the air gap between magnet and the rebar. Due to the physical clearance and the safety of the highpower magnet yoke, minimum distances was limited to 30mm. The air gap was varied from $30 \mathrm{~mm}$ to $75 \mathrm{~mm}$. After, $75 \mathrm{~m}$ we found week adhesion forces. Results obtained during the experiment was plotted against the FEA results to validate the modelling procedure.

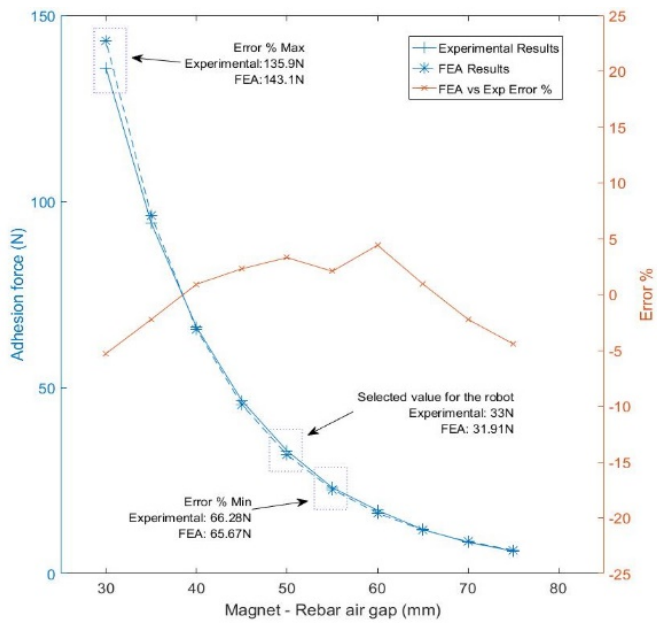

Figure 5: Comparison between experimental results and FEA from COMSOL

The above figure 5 compares the experimental results and the FEA results demonstrating a good agreement. However, $\approx 5 \%$ error was recorded when the air gap is $30 \mathrm{~mm}$ and the min error was recorded when the air gap is $40 \mathrm{~mm}$ (i.e min error of $0.91 \%$ ). Systematic errors can be introduced as the reason for the $\approx \pm 5$ error percentage (i.e. when considering the air gap vs forces $\pm 1 \mathrm{~mm}$ can influenced the adhesion forces significantly ). The above FEA vs experimental results provides evidence to validate the COMSOL model.
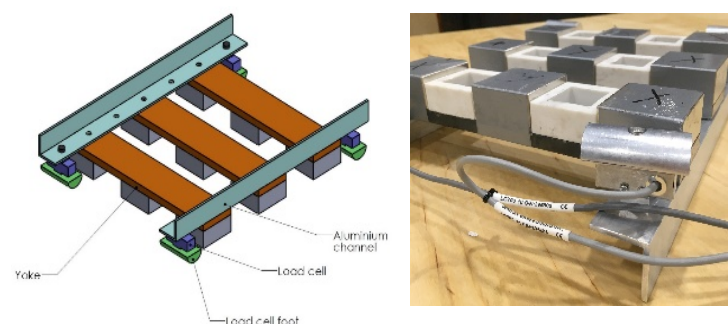

Figure 6: The 3 yoke adhesion module test rig. 
After the initial validation, a magnetic adhesion system consisting on 3 yokes systems was built and attached to an aluminum bar as shown in figure 6 . The total weight of this adhesion system was $\sim 10 \mathrm{~kg}$. Additionally, four OMEGA mini load sensors were intalled on each of the corners to measure the total force applied by the adhesion system.

\section{2. $\quad$ GPR module NDT testing}

A concrete test block (figure 7) was built containing different rebar thinning and various sizes of air voids, embedded at several known depths from the block surface. The PicoR-2K GPR was connected to a PC via USB-C cable to interface with the PicoR software version 4.3 in order to visualize the results.

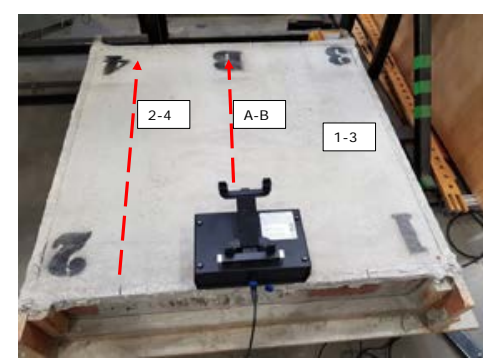

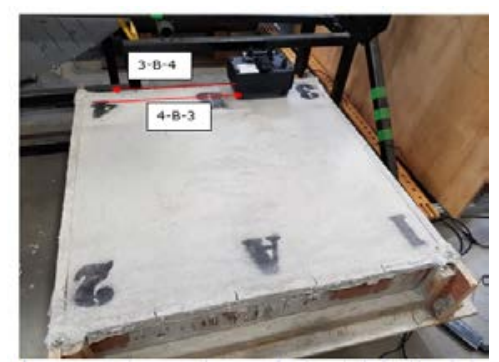

(b

Figure 7: (a) The three scanning paths used for detection of air voids. (b) Scan paths 3-B-4 and 4-B-3 used to detect both air voids and rebars

The following three scanning sequences were employed: (1) For air void detection: The GPR was moved in the direction of $1-3, A-B$ and $2-4$, as shown in figure $7(a)$. These paths were selected to avoid the rebars and to pass over the various voids within the concrete block; (2) For rebar detection: The GPR was moved in the direction of 1-A-2 and 3-B-4 (see figure 7(a)). These paths were selected to avoid the voids and to pass over the various rebar defects introduced; (3) For rebar and void detection: In this scanning sequence, the GPR was moved in the direction of 3-B-4 and then from 4-B-3, as shown in figure 7(b). The purpose of this trial was to evaluate the GPR with tightly spaced defects.

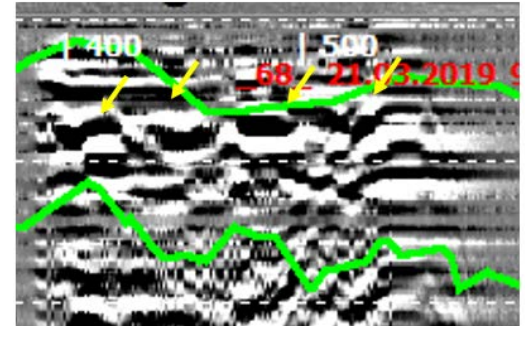

(a)

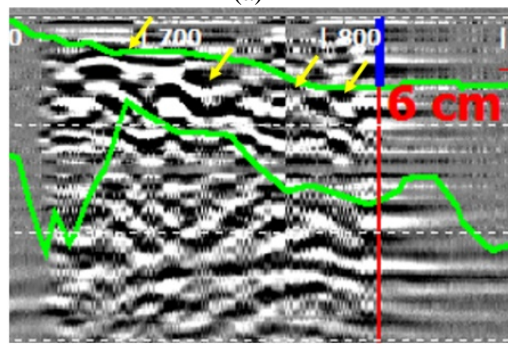

(c)

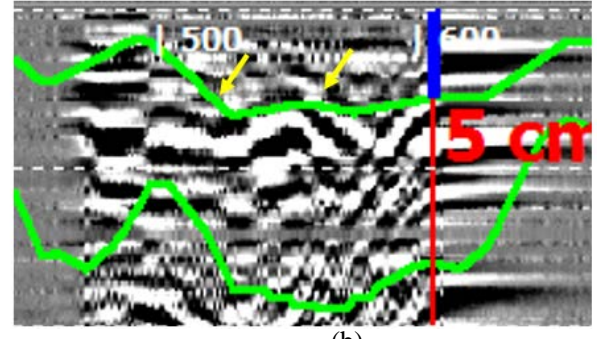

(b)

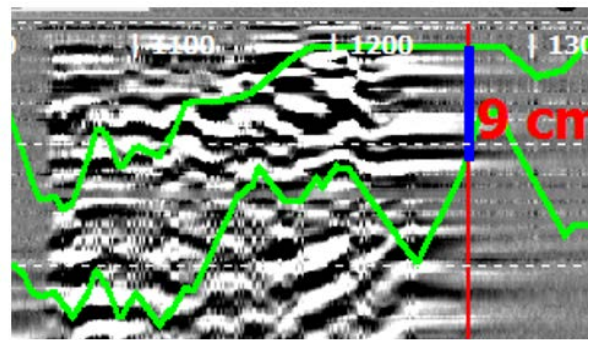

(d)

Figure 8: (a) Scan path A-B. (b) Scan path 2-4. (c) GPR detection of four rebars. (d). Scan path 4-B-3.

Sample results are shown in figure 8. To explain figure 8, (a) Scan path A-B: GPR signal showing detection of four air voids found in the line of the scan. The constant height of the peaks indicates the voids are at a similar depth. (b) Scan path 2-4 detecting two of the three 
defects in the line of the scan. (c) GPR scan showing detection of the four rebars in the concrete block. The downward trend of the peaks indicates that the rebars are positioned at increasing depths. (d). Scan path 4-B-3 showing detection of both rebars and air voids. The peak trend is upwards, which confirms that the scan moves from an area where the rebars and voids are at lower position to an area where the rebars and air voids are at a higher position. The capability of the PicoR-2k GPR device to detect the defects of interest was validated.

\subsection{Robot final prototype}

The development of the final prototype was built according to design specifications provided in the previous sections. The physical structure of the prototyped robot is shown in figure 9 .

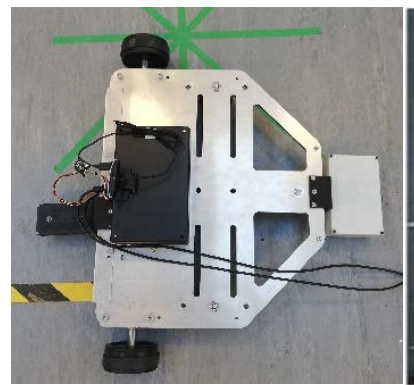

(a)

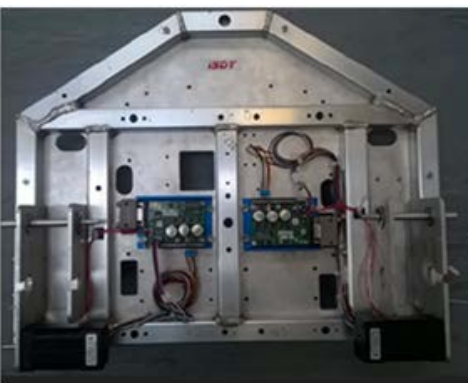

(b)

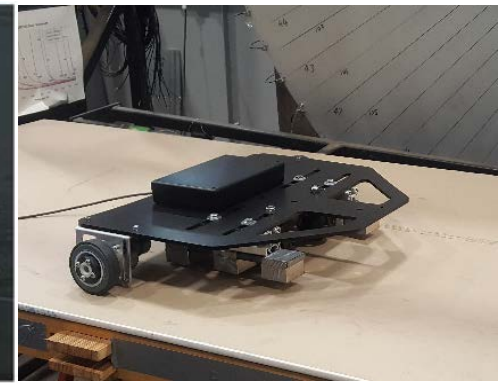

(c)

Figure 9: (a) Top side of the robot. (b) Bottom side without the adhesion module. (c) Fully prototyped crawler robot

\section{Testing}

A simulated climbing surface with the same rebar spacing as our testing concrete block, was created in the laboratory and several climbing tests were conducted as illustrated in the figure 10 .

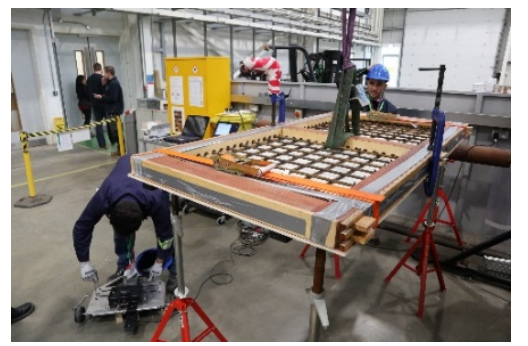

(a)

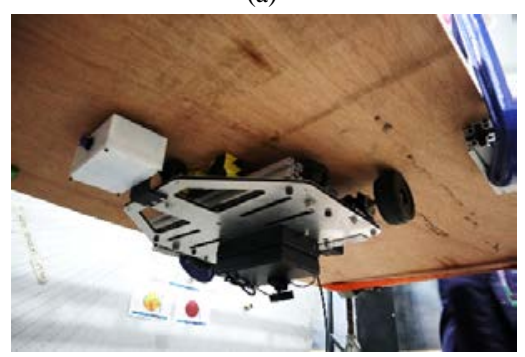

(b)

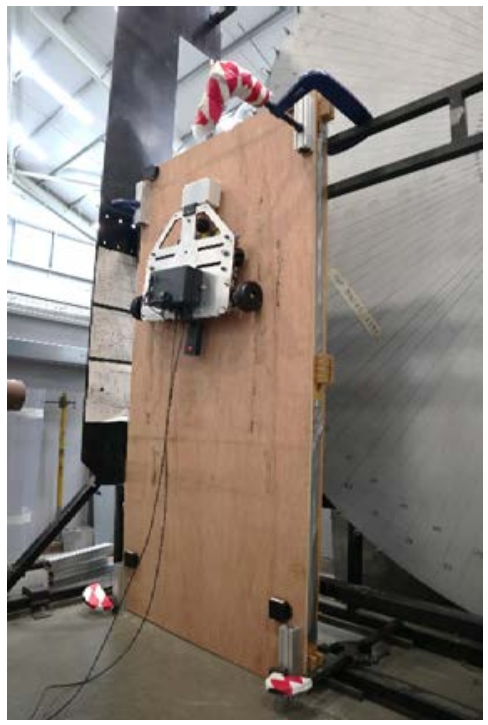

(c)

Figure 10: (a) Protype of the rebar based climbing surface. (b) Horizontal upside down climbing / adhesion test. (c) vertical climbing / adhesion test.

Full experimental video is available at $\rightarrow$ www.youtube.com/watch? $v=n w V G V c n l y k 4$ 


\section{Conclusion}

The aim of the project was to introduce a light-weight, fast moving (no lifting equipment) reinforced concrete inspection robot system with the ability of easy deployment and retreat features. The entire structure of the robot was built with aluminum alloy and carbon fiber. It was necessary to make sure the structural integrity is in a correct position after the assembly. Therefore, series of structural analysis experiments were carried out by using SolidWorks CAD design software and COMSOL software for FEA. Neodymium magnets (N52) are used in this robot to create the optimum addition force as per COMSOL simulations. In addition, a rebar detection and inspection system was designed using a GPR device. Finally, localization and performance of the final robot was tested. According to the laboratory trial tests and payload stability tests, we were able to validate and conclude that our crawler-based robot for inspection of reinforced concrete structures approach was successful.

\section{Acknowledgments}

This is work was sponsored by an Innovate UK grant (No: 103668), with the following partnership (in UK): London South Bank University (LSBU/LSBIC), Innovative Technology \& Science Ltd (InnoTecUK) and TWI Ltd.

\section{References}

[1] R. A. Market, "Infrastructure Monitoring Market by Technology (Wired and Wireless), Offering (Hardware: Sensors, Data Acquisition Systems; Software \& Services), Vertical (Civil infrastructure, Energy), Application \& Geography - Global Forecast to 2023," Research and Market, 2018.

[2] Lucintel, "Growth Opportunities in the Global Inspection and Testing Equipment Market in Civil Engineering," 2013.

[3] M. f. Silva and T. J. Machado, "A Survey of Technologies and Applications for Climbing Robots Locomotion and Adhesion," 2010. [Online]. Available: https://www.intechopen.com/books/climbing-and-walking-robots/a-survey-oftechnologies-and-applications-for-climbing-robots-locomotion-and-adhesion.

[4] F. Manuel \& J. Tenreiro (2010). "A Survey of Technologies and Applications for Climbing Robots Locomotion and Adhesion", INTECH.

[5] M. O. F. Howlader and T. . P. Sattar, "Finite Element Analysis based Optimization of Magnetic Adhesion Module for Concrete Wall Climbing Robot," International Journal of Advanced Computer Science and Applications, vol. 6, no. 8, pp. 8-18, 2015.

[6] M. O. f. Howlader and T. P. Sattar, "Development of Magnetic Adhesion Based Climbing Robot for Non-Destructive Testing," in Computer Science and Electronic Engineering Conference (CEEC), University of Essex, UK, 2015.

[7] M. O. F. Howlader, "Development of a Wall Climbing Robot and Ground Penetrating Radar System for Non-Destructive Testing of Vertical Safety Critical Concrete Structures," PhD thesis -London South Bank University, London , 2016.

[8] M. Dissanayake, T. Sattar, T.-H. Gan , I. Pinson and S. Lowe, "Design and prototype of a magnetic adhesion tracked-wheel robotic platform for mooring chain inspection," Journal of Systems and Control Engineering(Proceedings of the Institution of Mechanical Engineers, Part I), vol. 232, no. 8, pp. 1063-1074, 2018.

[9] C. P. F. Ulricksen, "Application of impulse radar to civil engineering," Doctoral Thesis, Department of Engineering Geology, Lund University of Technology, Sweden, 1982.

[10] M. Forde, "Ground penetrating radar," Introduction to Nondestructive Evaluation Technologies for Bridges," in Transportation Research Board Pre-conference Workshop, 2004. 